\title{
Retard mental avec $X$ fragile : une empreinte génomique très localisée, étroitement liée à l'expression clinique
}

Un pas décisif [1] vient d'être accompli, concernant l'analyse moléculaire et la compréhension du retard mental avec $\mathrm{X}$ fragile, la plus fréquente des maladies liées au chromosome $\mathrm{X}$ (un garçon atteint sur 1 500) [2]. La " course au gène" menée par un nombre croissant de laboratoires, selon les stratégies de la "génétique inverse " [2, 3] approche de son terme. Mais, surtout, les résultats obtenus dans notre laboratoire et rapportés par Vincent et al. [1] indiquent qu'une modification épigénétique très localisée de l'ADN (empreinte génomique par méthylation) joue un rôle capital dans l'expression de la maladie et laissent entrevoir de nouvelles possibilités de diagnostic moléculaire de la maladie, qui pourraient remplacer la délicate analyse cytogénétique.

Notre équipe était engagée depuis 1983 dans la "pêche " et la caractérisation de sondes proches du site fragile, localisées par cartographie génétique (études de liaison de marqueurs polymorphiques dans des familles) et cartographie physique (utilisation de panel de cellules comportant des cassures du chromosome $\mathrm{X}$ dans la région Xq27-q28, électrophorèse en champ pulsé) (pour une revue des stratégies utilisées, voir [2, 3]). C'est ainsi que plusieurs sondes polymorphiques ont été récemment isolées et localisées dans les deux régions qui apparaissaient les plus proches du locus $\mathrm{X}$ fragile [4]. L'une d'entre elles, Do33 (DXS465), a été obtenue par la combinaison de deux techniques récentes: l'amplification de séquences inter Alu (Alu-PCR) réalisée à partir d'un hybride somatique "réduit" par irradiation aux rayons $\mathrm{X}$. Cette sonde a été utilisée par Vincent et al. dans des expériences de cartographie de grands fragments de restriction, par électropho-

comparer l'organisation de la région chez des sujets normaux et des patients avec X fragile. Des différences spectaculaires ont été observées en utilisant les enzymes de restriction BssHII, SacII et EagI. La sonde Do33 détecte un fragment BssHII de 620 kilobases chez les sujets normaux. Le fragment est absent chez la grande majorité des 21 patients (mâles) examinés, remplacé par un fragment de taille supérieure à 1500 kilobases. Cela aurait pu suggérer l'existence d'un réarrangement génomique ( très grande insertion ou délétion d'un segment contenant un site BssHII). Toutefois, cette interprétation est contredite par le fait que certains patients ont un fragment de $620 \mathrm{~kb}$ présent, mais avec un dosage très diminué, et surtout quatre mâles normaux transmetteurs (mâles dont l'analyse familiale permet de démontrer qu'ils portent la mutation, mais qui n'ont pas d'expression clinique ou cytogénétique) montrent la présence d'un fragment de taille et d'intensité normales. L'anomalie détectée ne peut donc pas correspondre à la mutation. Des observations similaires ont été faites pour les enzymes SacII et EagI. Or les trois enzymes utilisées comportent des dinucléotides $\mathrm{CpG}$ dans leur site de reconnaissance et sont inhibées par la présence de cytosine méthylée au niveau de ce site (ces sites sont par ailleurs rencontrés préférentiellement dans les "îlots CpG ", qui marquent souvent l'extrémité 5' de gènes) [5]. Les anomalies observées peuvent donc s'expliquer par la méthylation d'un ilôt CpG proche de la sonde Do33, étroitement liée à l'expression phénotypique de la maladie. Il faut rappeler en outre que les îlots $\mathrm{CpG}$, présents sur le chromosome $\mathrm{X}$, sont non méthylés sur le chromosome $\mathrm{X}$ actif, mais sont méthylés sur le $X$ inactif [6]. La méthylation observée chez les patients pourrait donc s'interpréter comme une inactivation localisée du chromosome X. Cela est en accord avec une hypothèse très séduisante élaborée à partir de 1987 par Charles Laird [7, 8], un généticien de drosophile de Seattle (WA, USA), hypothèse qui n'avait pas reçu jusqu'ici de confirmation indépendante.

Afin d'expliquer les anomalies de ségrégation caractéristiques du syndrome $\mathrm{X}$ fragile [2, 9], Laird avait proposé que la mutation $\mathrm{X}$ fragile qui agit en cis bloque localement la réactivation d'un chromosome $\mathrm{X}$ précédemment inactif (une femme possède un $\mathrm{X}$ actif et un $\mathrm{X}$ inactif, mais préalablement à l'oogenèse, le $\mathrm{X}$ inactif est réactivé). L'expression de la maladie ne serait obtenue que si le chromosome muté passe par un cycle d'inactivation et de réactivation incomplète, entraînant une altération épigénétique (empreinte génomique ou tatouage). Un mâle normal transmetteur serait donc porteur d'un $\mathrm{X}$ non tatoué, ainsi que ses filles vectrices obligatoires de la mutation. A la génération suivante, au contraire, l'expression cytogénétique et clinique est réalisée chez les enfants ayant hérité de la mutation sur un chromosome " tatoué " (précédemment inactif)*. En combinant les résultats de Vincent et al., et l'hypothèse de Laird, on peut proposer le schéma suivant, qui reste cependant à démontrer.

Le site fragile serait, selon Laird

\footnotetext{
* "En fait, selon celle hypothèse, seulement $50 \%$ des mâles porteurs de la mulation seraient cliniquement alteints, ce qui contraste avec la pénétrance de $80 \%$ déduile des éludes épidémiologiques [9]. Laird, dans un argument très élaboré [8], propose que celle contradiction apparente serait due à l'existence de seulement deux cellules progénitrices des oogonies, au moment où l'inactivation du X est élablie, ainsi qu'à un biais d'observation (les fratries comportant des máles normaux transmetleurs mais pas de máles cliniquement alteints n'élant en général pas éludiées).
} 
[10], une région à réplication tardive (comme l'est le chromosome $\mathrm{X}$ inac-

$\mathrm{X}$, la méthylation, et la constitution du site fragile, sera d'un très grand

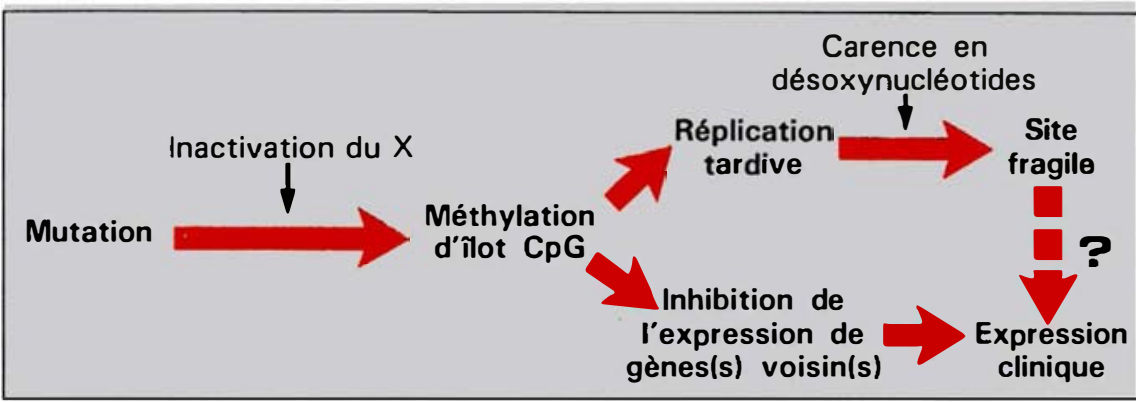

tif). La carence en désoxynucléotides imposée par le milieu de culture entraînerait, dans certaines cellules, une réplication incomplète et une anomalie de condensation de la chromatine (le site fragile). Le phénotype clinique serait dû à l'inhibition du ou des gènes flanquant l'îlot $\mathrm{CpG}$ méthylé. Toutefois, il est également possible que l'expression in vivo du site fragile (dans des cellules ayant un pool limitant de désoxynucléotides) pourrait participer à l'expression clinique comme suggéré par Warren [11]. La région qui contient les sites $\mathrm{CpG}$ anormalement méthylés a été récemment clonée dans notre laboratoire [12] sous forme de chromosome artificiel de levure (YAC) [13] par criblage de la banque de YACs construite au Centre d'étude du polymorphisme humain (CEPH) [14]. L'analyse par hybridation in situ confirme que ce clone YAC contient effectivement le site fragile (ainsi d'ailleurs que les séquences correspondant aux points de cassure chromosomiques engendrés par induction du site fragile $[2,15])$ et indique que la région de chromatine décondensée a la même position chez différents patients, et ne couvre pas plus de 100 à $200 \mathrm{~kb}$. L'utilisation des séquences clonées va permettre de rechercher et de caractériser les mutations à l'origine de la maladie (la haute fréquence du syndrome $\mathrm{X}$ fragile conduit à penser que le taux de mutation est encore plus élevé que pour la myopathie de Duchenne, et il pourrait s'agir d'une région très instable), et d'identifier le ou les gènes impliqués dans l'expression clinique. L'étude des mécanismes moléculaires reliant la mutation, l'inhibition supposée de la réactivation du intérêt fondamental, mais risque d'être longue. Par ailleurs, il est parfaitement envisageable d'utiliser des sondes de l'îlot $\mathrm{CpG}$ pour détecter, par Southern blot, la méthylation des sites de restriction et diagnostiquer très simplement la maladie. Il faut rappeler en effet que l'analyse cytogénétique est très délicate et lourde, car il faut des conditions de culture spéciales, l'analyse d'un grand nombre de mitoses, etc. [2]. Une méthode peu coûteuse et rapide permettrait un diagnostic plus précoce et plus systématique chez des enfants atteints de retard mental et donc un conseil génétique plus efficace. Sur le plan du diagnostic prénatal, ces nouvelles possibilités apporteront peutêtre un éclairage nouveau à un problème éthique difficile. En effet, il est actuellement possible de savoir, grâce à une étude familiale utilisant des marqueurs polymorphiques, si un fotus (mâle ou femelle) a hérité de la mutation. Or seuls $80 \%$ des garçons et $30 \%$ des filles porteurs de cette mutation ont un retard mental (moins marqué chez les filles). Il n'existe jusqu'ici aucune possibilité de prédiction, et notamment pour les filles se pose, de manière délicate, le problème d'une éventuelle interruption de grossesse face à ce risque de $30 \%$. Si les anomalies de méthylation sont retrouvées au niveau de l'ADN de villosités choriales, cela pourrait fournir une base prédictive quant à l'expression clinique, qui serait fondée toutefois uniquement sur des études rétrospectives et non sur des études prospectives.

De nombreuses équipes travaillent actuellement sur les aspects moléculaires du retard mental avec $X$ fragile, et disposent également de clo- nes YAC. On peut s'attendre, dans les prochains mois, à des nouvelles importantes, et peut-être surprenantes, concernant ce syndrome.

\section{Isabelle Oberlé \\ Jean-Louis Mandel \\ Cnrs et Inserm U. 184, Institut de chi- mie biologique, 11, rue Human, 67085 Strasbourg Cedex, France.}

1. Vincent A, Heitz D, Petit C, Kretz C, Oberlé I, Mandel JL. Abnormal pattern detected in fragile $X$ patients by pulsed field gel electrophoresis. Nature 1991 ; 349 : 624-6. 2. Jordan BR, Mattei JF. Retard mental lié à la fragilité du chromosome $X$ : où en eston en 1989 ? médecine/sciences $1989 ; 5: 450-8$. 3. Jordan BR. Chroniques génomiques. Le programme génome humain et le retard mental lié à la fragilité du chromosome $\mathrm{X}$. La microdissection revient en force et les YACs avancent. Et ensuite ? médecine/sciences $1990 ; 6$ : 157-9.

4. Rousseau F, Vincent A, Rivella S, et al. Four chromosomal breakpoints and four new probes mark out a 10-cM region encompassing the fragile-X locus (FRAXA). Am J Hum Genet 1991 ; 48 : 108-16.

5. Jordan BR. Ilots HTF : le gène annoncé. médecine/sciences 1991; $7: 163$.

6. Grant SG, Chapman VM. Mechanisms of $\mathrm{X}$ chromosome regulation. Ann Rev Genet $1988 ; 22$ : 199-234.

7. Laird CD. Proposed mechanism of inheritance and expression of the human fragile- $X$ syndrome of mental retardation. Genetics 1987 ; 117 : 587-99.

8. Laird CD, Lamb MM, Thorne JL. Two progenitor cells for human oogonia inferred from pedigree data and the $\mathrm{X}$-inactivation imprinting model of the fragile-X syndrome. Am J Hum Genet 1990 ; 46 : 696-719.

9. Sherman SL, Jacobs PA, Morton NE, Froster-Iskenius U, et al. Further segregation analysis of the fragile $X$ syndrome with special reference to transmitting males. Hum Genet $1985 ; 69$ : 289-99.

10. Laird C, Jaffe E, Karpen G, Lamb M, Nelson R. Fragile sites in human chromosomes as regions of late-replicating DNA. Trends Genet 1987 ; 3 : 274-81

11. Warren ST. Fragile $X$ syndrome : a hypothesis regarding the molecular mechanism of the phenotype. Am J Med Genet 1988; 30 : 681-8.

12. Heitz D, Rousseau F, Devys D, et al. Isolation of normal sequences that span the fragile $\mathrm{X}$ site and identification of a $\mathrm{CpG}_{\mathrm{p}}$ island involved in fragile X expression. Science 1991; 251: 1236-9.

13. Jordan BR. La montée en puissance des YAC. médecine/sciences $1990 ; 6: 470-2$.

14. Albertsen HM Abderrahim $\mathrm{H}$ Cann HM, Dausset J, Le Paslier D, Cohen D. Construction and characterization of a yeast artificial chromosome library containing seven haploid human genome equivalents. Proc Natl Acad Sci USA $1990 ; 87$ : 4256-60.

15. Warren ST, Knight SJL, Peters JF, Stayton $\mathrm{CL}$, Consalez GG, Zhang F. Isolation of the human chromosomal band $\mathrm{Xq} 28$ within somatic cells hybrids by fragile $\mathrm{X}$ site breakage. Proc Natl Acad Sci USA 1990 ; 87 : 3856-60. 